\title{
Correlation reflectometry at TEXTOR
}

A. Krämer-Flecken, S. Soldatov, B. Vowinkel, and P. Müller

Citation: Review of Scientific Instruments 81, 113502 (2010);

View online: https://doi.org/10.1063/1.3497305

View Table of Contents: http://aip.scitation.org/toc/rsi/81/11

Published by the American Institute of Physics

\section{Articles you may be interested in}

Plasma transport coefficients for nonsymmetric toroidal confinement systems

The Physics of Fluids 29, 2951 (1998); 10.1063/1.865495

Low noise $\mathrm{K}_{\alpha}$-band hopping reflectometer based on yttrium iron garnet sources at TEXTOR Review of Scientific Instruments 82, 033513 (2011); 10.1063/1.3567779

Multi-channel poloidal correlation reflectometry on experimental advanced superconducting tokamak Review of Scientific Instruments 87, 11E707 (2016); 10.1063/1.4960162

Implementation of the new multichannel $\mathrm{X}$-mode edge density profile reflectometer for the ICRF antenna on ASDEX Upgrade

Review of Scientific Instruments 87, 11E722 (2016); 10.1063/1.4961558

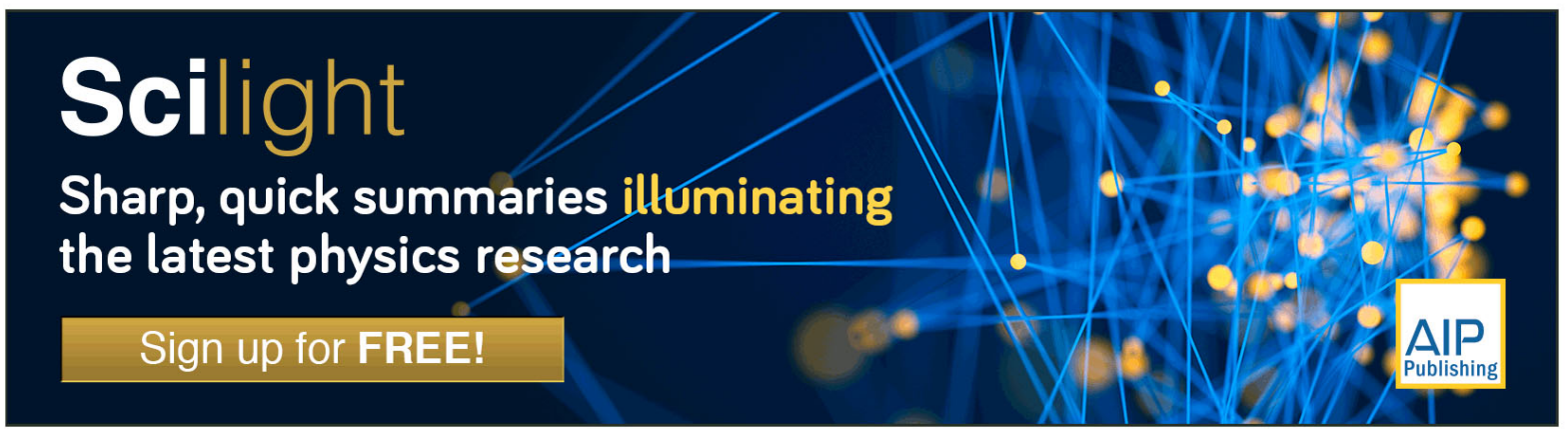




\title{
Correlation reflectometry at TEXTOR
}

\author{
A. Krämer-Flecken, ${ }^{1, a)}$ S. Soldatov, ${ }^{2}$ B. Vowinkel, ${ }^{3}$ and P. Müller ${ }^{4}$ \\ ${ }^{1}$ Institute for Energy Research (Plasma Physics), Forschungszentrum Jülich, EURATOM Association, \\ D-52425 Jülich, Germany \\ ${ }^{2}$ Department of Applied Physics, Ghent University, Rozier 44, 9000 Ghent, Belgium \\ ${ }^{3}$ 1. Physikalisches Institut, Universität zu Köln, Zülpicher Strasse 77, 50937 Köln, Germany \\ ${ }^{4}$ Argelander-Institut für Astronomie, Auf dem Hügel 71, 53121 Bonn, Germany
}

(Received 8 July 2010; accepted 13 September 2010; published online 9 November 2010)

\begin{abstract}
In high temperature fusion plasmas the transport of energy and particles is commonly believed to be driven by turbulence. Turbulence quantities as correlation length and decorrelation time are important for the confinement properties of a plasma. Besides other diagnostics, correlation reflectometry has proven to be a suitable tool for the measurement of turbulence properties. At the medium sized Toroidal EXperiment for Technical Oriented Research (TEXTOR) the existing correlation reflectometry has been recently upgraded. A new reflectometer based on a microwave synthesizer has been developed and installed for the investigation of turbulence properties in a fusion plasma. Together with the existing reflectometer the measurement of radial correlation length and decorrelation time becomes available. Both reflectometers are computer controlled and allow to program individual frequency sequences and the duration of each frequency step. With the existing poloidal antenna array at $\theta=0^{\circ}$ and on top of the vacuum vessel, the system allows the measurement of radial correlation and poloidal correlations at the same time. First experiments have been performed and the results on the radial correlation length of density fluctuations in a fusion plasma are presented. [doi:10.1063/1.3497305]
\end{abstract}

\section{INTRODUCTION}

High temperature fusion plasmas are produced in either stellarators ${ }^{1}$ or tokamaks. ${ }^{2}$ In these devices the plasma is confined by magnetic fields. The superposition of toroidal and poloidal magnetic fields produces eccentrical flux surfaces where, in first approximation, electron temperature and density are constant. One of the urgent tasks in magnetic confined fusion plasma physics is the understanding of the origin of the anomalous radial transport. It is commonly believed that small scale turbulence, e.g., density fluctuation, is responsible for the radial transport. In a fusion plasma small scale structures are aligned along magnetic field lines. Especially, the interaction of small scale and mesoscale structures and their contribution to transport of energy and particles on one side and the generation of transport barriers ${ }^{3}$ on the other side is of interest for the design and operation of large tokamaks as ITER. ${ }^{4}$ The properties of these fluctuations can provide new insight into transport mechanisms. However, the observation of fast turbulent processes is difficult and needs high temporally and spatially diagnostics. One of them, the reflectometry, is operated in the range of millimeter waves and sensitive to fluctuations in the electron density. It makes use of the optical properties of a high temperature plasma. The refraction index decides between regions of absorption, transmission, and reflection. Choosing a frequency range which allows only for reflection at a certain isodensity surface, density fluctuations near this reflection layer can be related to amplitude and phase fluctuations of the probing

${ }^{a)}$ Electronic mail: a.kraemer-flecken@fz-juelich.de. frequency. Combining different antennas in an array allows to study spatial correlations and the propagation of turbulent structures. The capability of correlation reflectometry has been discussed by Rhodes and co-workers. ${ }^{5}$ Since then it is used for the estimation of correlation length and decorrelation time at the same flux surface. ${ }^{6}$ With an additional reflectometer operating at a slightly different frequency the radial correlation length can be estimated as demonstrated at JET. ${ }^{7,8}$ This quantity is of importance for the radial electron transport. In addition, correlation reflectometry for $\mathrm{O}$ - and $\mathrm{X}$-mode is used for the estimation of the local magnetic field. ${ }^{9,10}$

The paper is organized as follows. In Sec. II the instrumentation is discussed with emphasis on the new generator. Also the antenna arrays installed in the tokamak are described in detail and especial attention is paid to the arrangement of the antennas in the array to deduce the inclination angle of the magnetic field line. Section III explains the different possible correlation schemes available at TEXTOR and in Sec. IV, first measurements of long range correlations and the measurement of the radial correlation length are presented. Section V summarizes the results.

\section{PRINCIPLES AND INSTRUMENTATION}

\section{A. Conditions for reflection}

The condition for absorption and reflection of waves in the plasma is governed by the refractive index $(N)$, which is given by the Appleton-Hartree equation. ${ }^{11}$ For the probing 


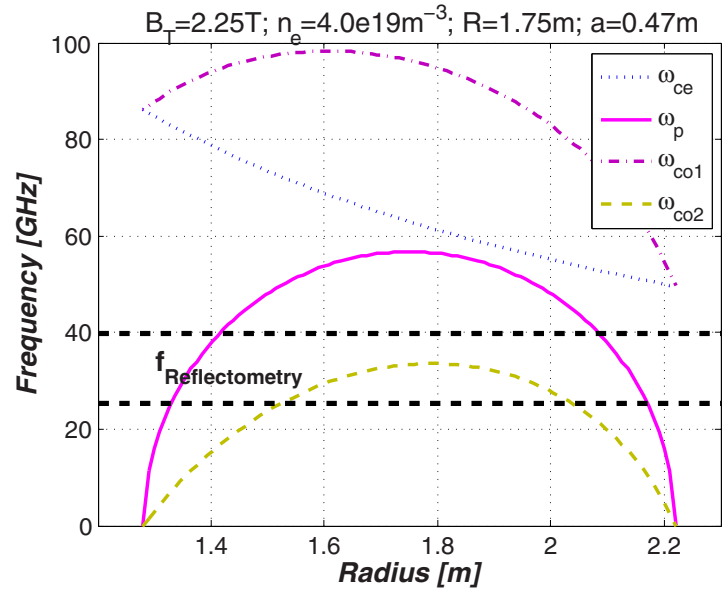

FIG. 1. (Color online) Plasma frequency $\omega_{p}$-solid, $\omega_{c e}$-dotted, upper X-mode cutoff $\omega_{\mathrm{col}}$-dashed, and lower X-mode cutoff $\omega_{\mathrm{co} 2}$ for a parabolic density profile with central $n_{e}=4 \times 10^{19} \mathrm{~m}^{-3}$. The dashed horizontal lines mark the frequency range of the reflectometry system.

frequency $\omega$, the condition for reflection $\left(N^{2}=1\right)$ and the assumption of a perpendicular viewing angle to the plasma, the equation can be simplified to

$$
\omega^{2}=\omega_{p}^{2}
$$

for O-mode propagation (the $E$-field vector is parallel to the magnetic field). Here the plasma frequency is denoted by $\omega_{p}=\sqrt{n_{e} e^{2} / \epsilon_{0} m_{e}}$ and depends only on the electron density $n_{e}$. In case of X-mode polarization, reflection occurs for the lower and upper X-mode cutoff, which is given by

$$
\omega_{R, L}^{2}=\frac{1}{2}\left[\left(2 \omega_{p}^{2}+\omega_{c}^{2}\right) \pm \omega_{c} \sqrt{4 \omega_{p}^{2}-\omega_{c}^{2}}\right] .
$$

Here cyclotron frequency is denoted by $\omega_{c}=e B / m_{e}$, where $B$ is the local magnetic field strength. Compared to O-mode polarization the use of the lower X-mode polarization allows to use the same frequency to probe a reflection layer deeper in the plasma.

At TEXTOR (Ref. 12) both polarizations are realized, but not for all antenna arrays used. The reflection layers for $\mathrm{O}$ - and X-mode polarization for TEXTOR parameters are shown in Fig. 1. In addition, the frequency range of the reflectometry system at TEXTOR is given by the horizontal dashed lines.

\section{B. Microwave generators}

At TEXTOR two microwave generators are operational. Both are operated in the so-called $\mathrm{K}_{a}$-band $(26-40 \mathrm{GHz})$ and each of them consists of two sources with frequencies $f_{1}$ and $f_{2}$, which are out of tune by $20 \mathrm{MHz}$. Both sources are phase coupled by a phase locked loop (PLL). Whereas one generator $(26 \leq f \leq 37 \mathrm{GHz})$ uses low noise yttrium-iron-garnet (YIG) tuned oscillators which can be swept only slowly with $10 \mathrm{GHz} / \mathrm{s}$, the second one $(24 \leq f \leq 40 \mathrm{GHz})$ allows a fast frequency change within $\delta t \leq 1 \mathrm{~ms}$ for an arbitrary frequency step. For achieving the fast frequency change and enough output power $\left(P_{\text {out }} \leq 40 \mathrm{~mW}\right)$ voltage controlled oscillators (VCOs) are used. Such oscillators, having enough power, are not available in the $\mathrm{K}_{a}$-band. Therefore oscillators at a lower frequency have to be used and multiplied in frequency to cover the frequency range from 24 to $40 \mathrm{GHz}$. However, each frequency doubling reduces the signal strength of the output signal and amplifiers have to be used to increase the signal strength again. The frequency range and power of the oscillator are selected carefully to match the specifications of the output signal in the $\mathrm{K}_{a}$-band and to provide a low phase noise. A sketch of the generator is seen in Fig. 2. In the case of TEXTOR reflectometry it was de-

\section{Dual Frequency Synthesizer $24-40 \mathrm{GHz}$}

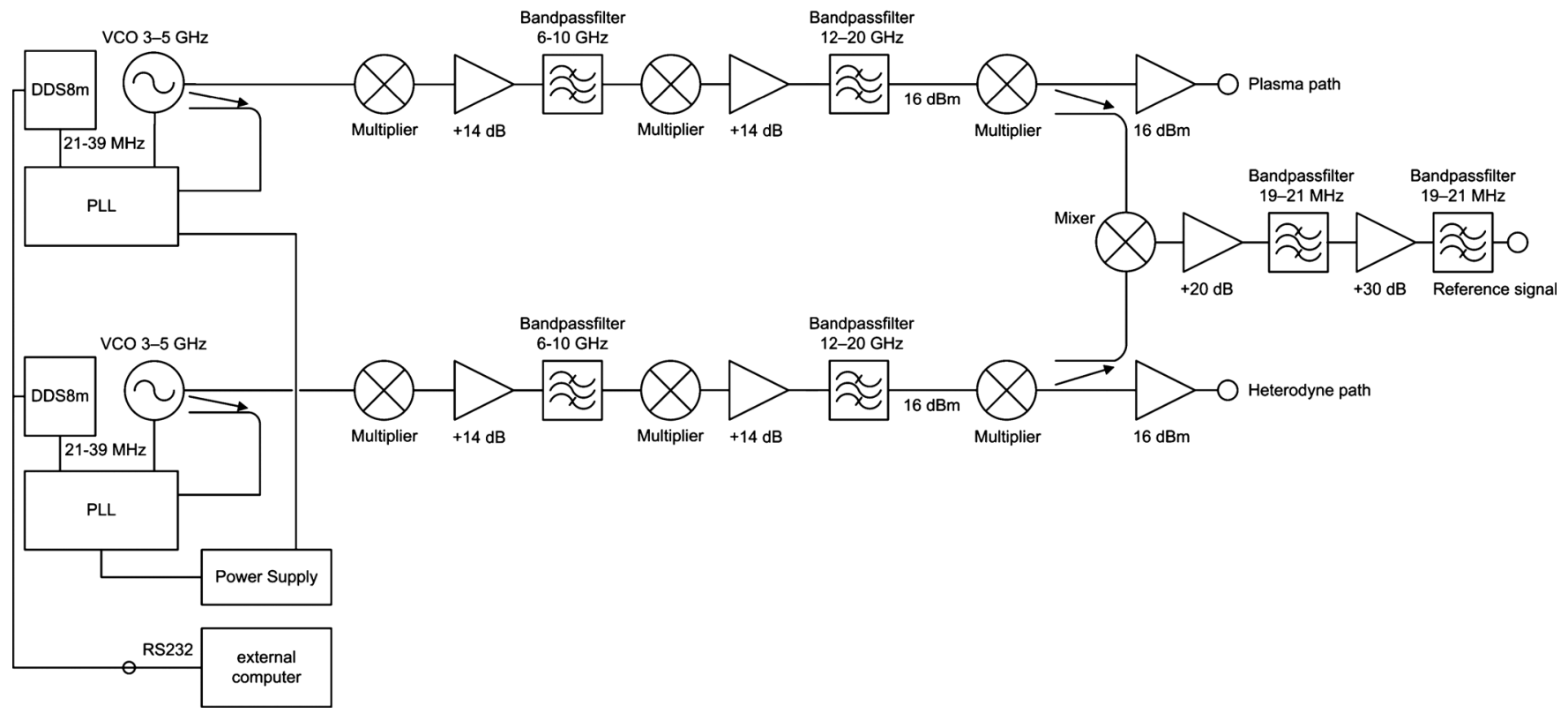

FIG. 2. Setup of the new reflectometer generator with two VCOs and PLL. To achieve the $\mathrm{K}_{a}$-band frequencies multiplying of the VCO frequency is necessary. Each multiplier increases the frequency by a factor of 2 . 


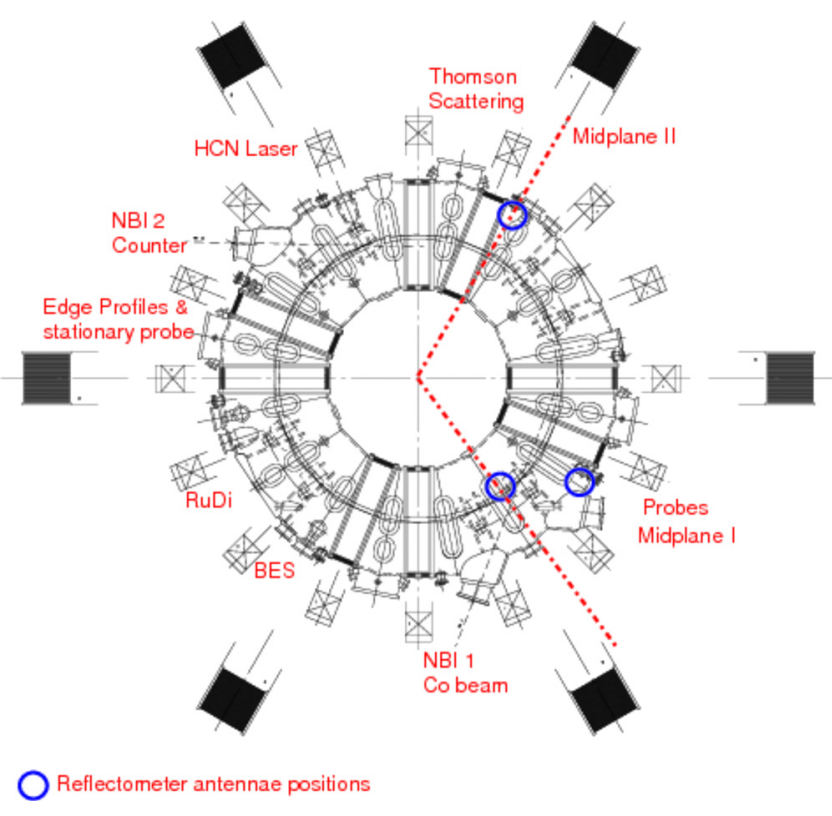

FIG. 3. (Color online) The position of the three antenna systems installed at TEXTOR. In addition, the location of different diagnostics is visible.

cided to use VCOs in the range of 3-5 GHz. With a frequency multiplication of 8 it covers the full $\mathrm{K}_{a}$-band. Besides the frequencies $f_{1,2}$ the reference frequency $f_{\text {ref }}=f_{1}-f_{2}$ $=20 \mathrm{MHz}$ signal is provided from both VCOs and used later for down conversion in the quadrature detectors (see Sec. II E). This $20 \mathrm{MHz}$ signal is only available when both frequencies are phase locked.

Both generators also have a serial interface which allows to program the frequency of each oscillator and the time duration of the frequency step. Whereas the control software for the reflectometer with the YIG-oscillator allows up to 20 different frequency steps, each at the same duration, the VCO based reflectometer can be programmed for eight different time windows and frequency steps individually. In this reflectometer the time window range should be $\leq 10000 \mathrm{~ms}$. The time step itself is programmed in milliseconds. Two trigger inputs allow arming of the system as well as starting the preprogrammed frequency sequence. After one sequence the system automatically falls back into the program modus. The VCO based reflectometer can be triggered several times during a discharge, whereas the YIG based reflectometer needs a substantial delay (several seconds) for recalibration after a sweep.

\section{Antennas and waveguides}

At TEXTOR three antenna arrays are installed (see Fig. 3 ). The circles denote the locations of the antenna array. Two of them are in the equatorial plane at the outer midplane, denoted as midplane I and midplane II, the third one on top of the toroidal vessel. With respect to the top array the other two arrays are installed at $\Delta \theta \geq 90^{\circ}, \Delta \phi=22.5^{\circ}$ and $\Delta \theta$ $\geq 90^{\circ}, \Delta \phi=112.5^{\circ}$. The fact that $\Delta \theta \geq 90^{\circ}$ stems from a shift of the top antenna array toward the high field side by $s$ $=0.08 \mathrm{~m}$ with respect to the major radius $R_{0}$. Furthermore, due to the Shafranov shift the angle increases with decreasing reflection radius. All three arrays are different in the

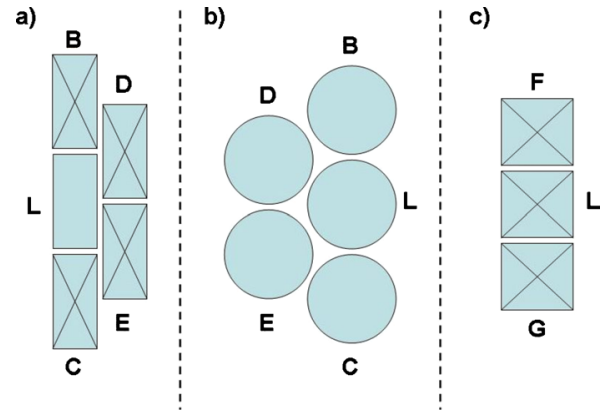

FIG. 4. (Color online) Different antenna arrays used at TEXTOR: (a) top array, (b) midplane array I, and (c) midplane array II. Note that L denotes the launcher antenna.

number of receiving antennas and in the shape of a single antenna. All arrays are installed $5 \mathrm{~mm}$ behind the liner. The liner radius amounts to $r_{\text {liner }}=546 \mathrm{~mm}$. The two arrays, which are separated by $\Delta \phi=22.5^{\circ}$, consist of one launching antenna and four receiving antennas. They are grouped in the array as shown in Fig. 4(a). The top one uses pyramidal horn antennas with a focal length of $l=93 \mathrm{~mm}$. The antenna mouth is rectangular $(49 \times 32 \mathrm{~mm})$. The antenna foot ends in a rectangular $\mathrm{K}_{a}$-band fundamental WR28 rectangular waveguide made of copper. The same waveguide is also used to guide the microwaves outside the vessel to the measuring cabin. All five antennas aim to the plasma center. The geometrical poloidal distance between the antennas is $\Delta \theta_{\text {geo }}$ $=0.05,0.09,0.14,0.19 \mathrm{rad}$. The geometrical toroidal separation between the two antenna columns is $\Delta \phi=0.019 \mathrm{rad}$. The array is installed with the long antenna edge along the poloidal angle. This allows the propagation of the fundamental mode in rectangular waveguide, which means measurement in O-mode only.

The antenna array [see Fig. 4(b)] in the equatorial plane consists of the same antenna arrangement; however, each antenna has a circular mouth $d=50 \mathrm{~mm}$ in diameter and a focal length of $l=86 \mathrm{~mm}$. Due to the circular antenna mouth, the toroidal separation of the two columns is $43 \mathrm{~mm}$, which corresponds to $\Delta \phi=0.019 \mathrm{rad}$. Also this array aims to the plasma center which yields the same poloidal separation as for the top array. The antenna foot ends in a copper circular waveguide. The waveguide with an inner diameter of 8 $\mathrm{mm}$ is used in the vessel as well as outside the vessel on the way to the measurement cabin. The coupling of the circular waveguide to the WR28 rectangular ones is realized by smooth tapers with length of $50 \mathrm{~mm}$. The third antenna array consists of three antennas [see Fig. 4(c)]. Here the antenna mouth is quadratic with an edge of $40 \mathrm{~mm}$. The focal length is $l=75 \mathrm{~mm}$. The third array is constructed to measure Oand X-mode polarity of the reflected wave (see Sec. II A). The antenna in the middle is used for launching the wave, and the poloidal separation between the upper and lower antennas amounts to $\Delta \theta=0.71 \mathrm{rad}$. Following the vacuum break, a taper together with a twist selects the polarization of the reflected radiation. Turning the taper by $90^{\circ}$ and removing the twist will allow measurements for X-mode polarization as well. Following the taper, the waveguide to the measurement cabin is made of WR28 rectangular waveguide.

All antennas in the three arrays are made of stainless 


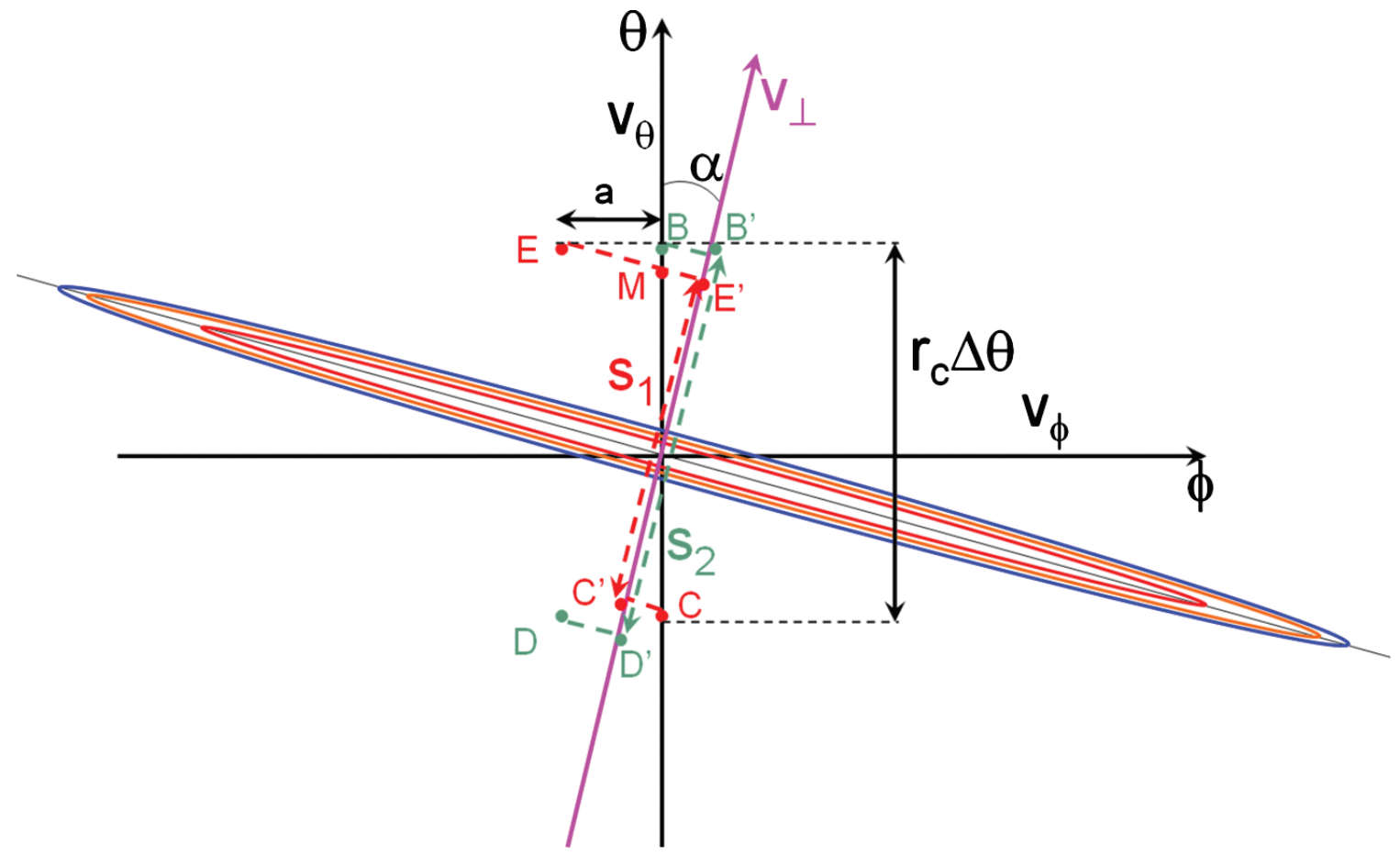

FIG. 5. (Color) Turbulence propagation direction for a case with $\alpha \neq 0$. The elliptical assumed eddy is elongated along the magnetic field line. It is clearly seen that the projection $\mathrm{EC}$ on $v_{\perp}$ given by $\mathrm{E}^{\prime} \mathrm{C}^{\prime}$ is smaller than the projection of $\mathrm{BD}\left(\mathrm{B}^{\prime} \mathrm{D}^{\prime}\right)$.

steel. The reflected microwave passes a vacuum window in the fundamental waveguide, made by a Kapton sheet with a thickness of $0.8 \mathrm{~mm}$ and an attenuation of $0.8 \mathrm{~dB}$. The vacuum break acts also as electrical insulation from the vessel. The signals feed the balanced mixer after passing a manual microwave switch. The switch is used to select between midplane and top antennas for each antenna (see Sec. III). The heterodyne branch to the mixer is supplied directly by the generator and is distributed to the mixer via $3 \mathrm{~dB}$ directional couplers. The frequency in the heterodyne branch $\left(f_{\mathrm{HD}}\right)$ and the frequency in the launching path $\left(f_{\text {launch }}\right)$ fulfill the equation $f_{\mathrm{HD}}-f_{\text {launch }}=f_{\mathrm{IF}}=20 \mathrm{MHz}$, where $f_{\mathrm{IF}}$ denotes the intermediate frequency.

\section{Antenna arrangement}

The arrangement of the antenna in the top and midplane array allows to determine the inclination or pitch angle of the turbulence. Therefore, it is assumed that the eddies follow in first approach the magnetic field line and are elongated along the field line. In terms of wave number it is equivalent to $k_{\|} \ll k_{\perp}$. The calculation of the cross correlation for the six combinations results in four different poloidal and toroidal combinations. The slope of the calculated delay time as a function of the poloidal separation $(\Delta \theta)$ is a measure for the propagation of the turbulence perpendicular to the magnetic field line $\left(B_{\perp}\right)$. However, the projection of an antenna combination with the same poloidal separation and a toroidal angle $\Delta \phi \neq 0$ rad, e.g., EC and BD (see Fig. 5-note that antennas $\mathrm{E}$ and $\mathrm{C}$ are poloidally shifted by $\Delta \theta_{\mathrm{geo}}=0.14 \mathrm{rad}$ for a better comparison), on the direction of $B_{\perp}$ yields a difference in the propagation path if the inclination angle $\alpha$ $\neq 0 \mathrm{rad}$. This offers the possibility to determine the inclination angle of the eddies from those combinations. In this case the measured ratio in the delay time depends only on geometrical properties of the antenna array and the reflection radius $r_{c}$

$$
\frac{s_{\mathrm{EC}}}{s_{\mathrm{BD}}}=\frac{\Delta t_{\mathrm{EC}}}{\Delta t_{\mathrm{BD}}}=\frac{r_{c} \Delta \theta-a \cdot \tan \alpha}{r_{c} \Delta \theta+a \cdot \tan \alpha} .
$$

Here $a$ denotes half the toroidal distance between both antenna columns. Note that also other combinations can be used for the estimation of the pitch angle as long as one of the used combinations fulfills the condition $\Delta \phi \neq 0 \mathrm{rad}$.

The ratio of poloidal magnetic field $\left(B_{\theta}\right)$ and toroidal magnetic field $\left(B_{\phi}\right)$ is proportional to the pitch angle by the relation

$$
\frac{B_{\phi}}{B_{\theta}}=\frac{1}{\tan \alpha} \propto q .
$$

Measuring $\alpha$ allows to determine $B_{\theta}$, and therefore the local safety factor $q$ from correlation reflectometry. This offers a new and robust method for the safety factor estimation even at the edge, where diagnostics as polarimetry or motional Stark effect (MSE) have difficulties. Also the restriction to neutral beam heated plasmas as in the case of MSE is not necessary. The consequence of the pitch angle estimation results in more robust estimation of the turbulence propagation velocity $\left(v_{\perp}\right)$ by taking into account the different propagation path having the same poloidal and toroidal angular separation. An example for a pitch angle corrected estimation of $v_{\perp}$ is shown in Fig. 6 for midplane antenna array and a reflection layer of $r_{c}=0.325 \mathrm{~m}$. It shows $\Delta t$ as a function of $\Delta \theta$ for the uncorrected data and as a function of $\Delta \theta_{\perp}$ for the corrected data, where $\Delta \theta_{\perp}$ is defined as $\Delta \theta_{\perp}=s / r_{c}$. Here $s$ denotes the distance on the $B_{\perp}$ direction, traveled by the turbulence. However, the estimated velocity for the uncor- 


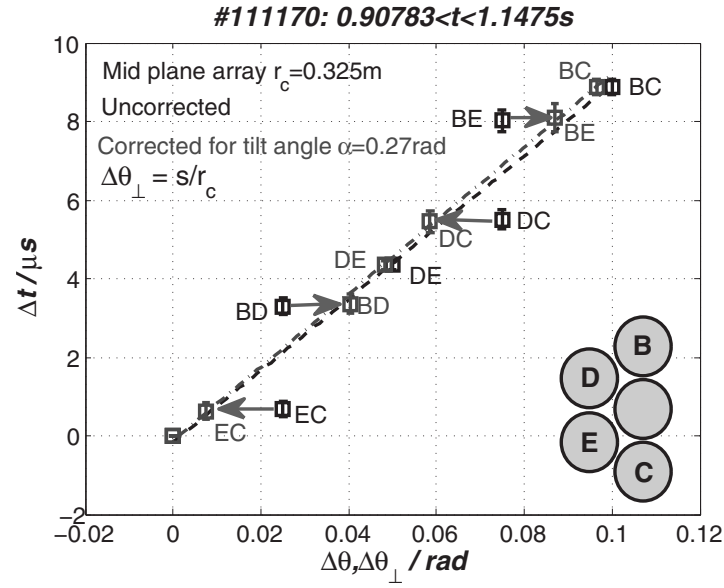

FIG. 6. Uncorrected and pitch angle corrected positions of the different antenna combinations. The dashed line represents for both cases the mean rotation, which is not much affected by the correction.

rected and the corrected data has not changed much due to the fact that the deviations in the uncorrected case are symmetric. Furthermore, the fact that the correction for the top array is smaller than for the midplane array is explained by an increased $B_{\phi}$. With the known pitch angle it is also possible to calculate the projection of $v_{\perp}$ on the $\theta$ axis, which makes the comparison with other diagnostics much simpler.

\section{E. Detection and acquisition}

The intermediate signal from the mixer $\left(f_{\mathrm{IF}}\right)$, belonging to one antenna, and the $20 \mathrm{MHz}$ reference signal from the generator $\left(f_{\text {ref }}\right)$ feed the quadrature detectors. The quadrature detectors are fabricated in house. They have a small bandwidth for the input signal. The amplitude of both signals together can be adjusted on the circuit board as well as the dc offset and the amplitude of the sine and cosine output signals. This allows an ideal matching to the input voltage range of the analog to digital converters (ADCs). Both signals are stored with a sampling rate of $1 \mathrm{MHz}$ for each antenna. The used $\mathrm{ADCs}$ are peripheral component interconnect (PCI) modules mounted in a PCI extension crate installed close to the quadrature detectors in the TEXTOR experimental area. Each ADC allows to store four signals with a resolution of 14 bit and 64 MSample on board memory for each channel. These parameters allow to store the complete pulse $\left(t_{\text {pulse }}\right.$ $\leq 10 \mathrm{~s}$ ) even with the highest sampling rate of $3 \mathrm{MHz}$. The short cabling reduces noise on the signals. The computer for storing the data is localized in an air conditioned server room outside the experimental area. The connection between the crate and the computer is done via an optical link.

\section{OPERATION SCHEMES}

The three antenna arrays are operated with two generators and allow different operation schemes, as can be seen in Fig. 7. It shows the receiving antenna of all three positions and the waveguide connections to both generators to illustrate the measurement arrangements. The use of manual microwave switches allows to select antennas from different arrays. The following measurement scenarios are possible:

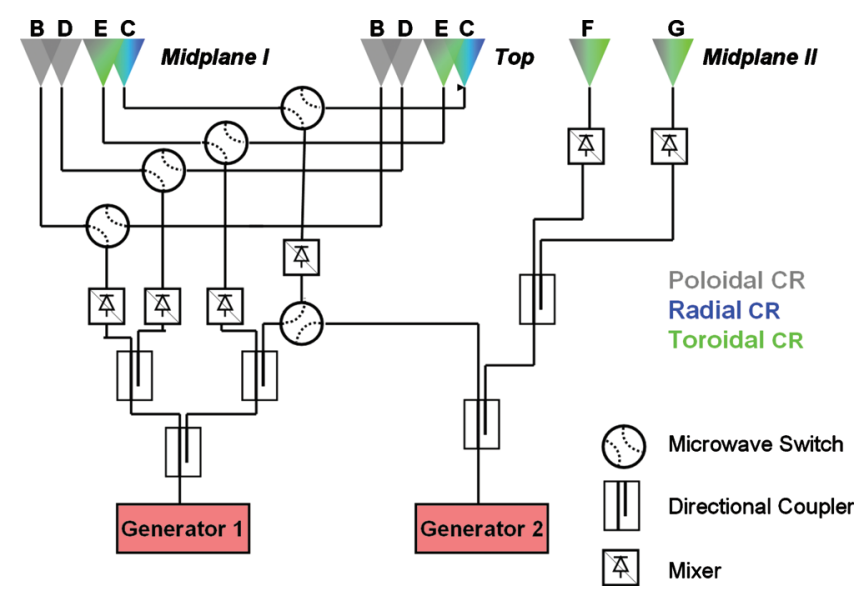

FIG. 7. (Color) Schematic presentation of the different correlation schemes at TEXTOR. Only the heterodyne path of the receiving antenna is shown. The different face colors of the antennas exemplify the possible correlations.

- Measurement of poloidal correlations either in the top or midplane arrays with one generator only allows the estimation of the rotation perpendicular to the magnetic field line as well as the estimation of the perpendicular correlation length $\left(l_{\perp}\right)$ and the decorrelation time $\left(\tau_{\mathrm{dc}}\right)$.

- Measurement of long distance correlations for the following separations:

(1) $\Delta \theta \geq 90^{\circ}, \Delta_{\phi}=22.5^{\circ}$;

(2) $\Delta \theta \geq 90^{\circ}, \Delta_{\phi}=112^{\circ}$; and

(3) $\Delta \theta=0^{\circ}, \Delta_{\phi}=90^{\circ}$.

- Measurement of radial correlations either with the top array or the midplane array. In both cases one antenna is connected to the second generator. In this scheme, radial and poloidal properties of the turbulence are measured as well as the rotation. In this scenario, it is possible to change the frequency of one generator only, or to change the frequency of both generators keeping the radial separation nearly constant. In the latter case the change in turbulence properties as a function of the radius can be investigated during a discharge.

The advantage of O-mode correlation reflectometry is the measurement of turbulence properties and the plasma rotation with the same diagnostic at the same time and radial position. This is of importance for long range correlation measurements. Furthermore, the measurement of $v_{\perp}$ allows to measure the radial electric field $E_{r}$, assuming the pressure gradient is known from density and temperature profiles. From the radial development of $v_{\perp}$ the rotation shear can be calculated. Both rotation shear and $E_{r}$ influence the turbulence properties and are discussed to play an important role in the generation of transport barriers.

\section{EXAMPLES OF CORRELATION MEASUREMENTS}

This section describes the first results on long range correlations and on radial correlation measurements at TEXTOR. Both examples are obtained by using O-mode polar- 


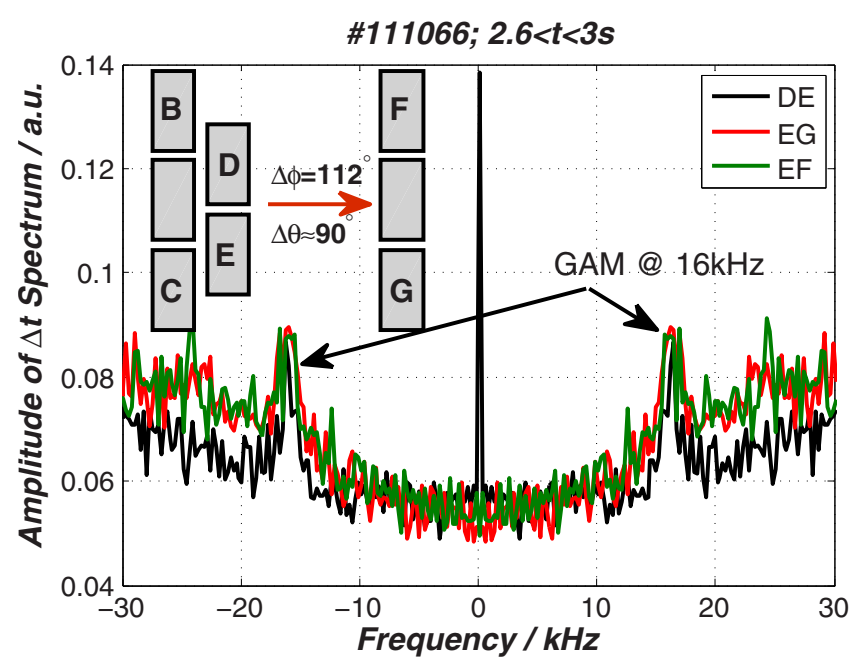

FIG. 8. (Color online) Verification of long range correlation in the $\Delta t$ spectrum for different antenna combinations. Antennas B, D, E, and C are on top and $\mathrm{F}$ and $\mathrm{G}$ at midplane. A clear peak at the GAM frequency is seen for all three combinations.

ization and highlight the application of the extended antenna array setup and the use of two reflectometers in a similar frequency band.

\section{A. Long range correlations}

For the investigation of long range correlation the combination between top array and midplane array with $\Delta \phi$ $=112^{\circ}, \Delta \theta \approx 90^{\circ}$ is used. The frequency of the first generator was set to $f_{1}=28 \mathrm{GHz}$, whereas the frequency of generator 2 was swept stepwise during the discharge $\left(25.3 \leq f_{2}\right.$ $\leq 30.2 \mathrm{GHz}$ ). The corresponding reflection layer is fixed to $r_{c} / a=0.83$ and $0.87 \leq r_{c} / a \leq 0.8$, respectively. In this radial range, geodesic acoustic modes (GAMs) ${ }^{13,14}$ are observed. This mode shows pronounced density fluctuations at the top and bottom of the vessel and has a poloidal and toroidal symmetric structure for velocity oscillations. As a consequence a correlation at the GAM frequency should be observed for the above toroidal and poloidal separation. Indeed, in Fig. 8 GAM frequency is seen in the delay time spectra of short (within the top antenna array combination DE) and long distance correlations (combinations of EF and EG). For the delay time spectrum the cross correlation for different antenna combinations is calculated. Each sample volume for the cross correlation is small, so that the Nyquist frequency of the spectrum is well above the GAM frequency. Note that the combination $\mathrm{DE}$ is calculated for $f_{1}=28 \mathrm{GHz}$ only, where the other combinations stem from different generators $\left(f_{1}=28 \mathrm{GHz}, f_{2}=28.1 \mathrm{GHz}\right)$. For the calculation of the $\Delta t$ spectrum, only the frequency range above $40 \mathrm{kHz}$ is used. The peak at the GAM frequency is therefore also evident for the modulation of the ambient turbulence rotation by the GAM.

\section{B. Radial correlations}

The radial correlation length and the radial propagation of turbulent eddies are studied for the top and midplane I antenna array separately. All analyzed discharges are Ohmic ones with $I_{p}=400 \mathrm{kA}$ and $B_{t}=1.9 \mathrm{~T}$. The line averaged den-

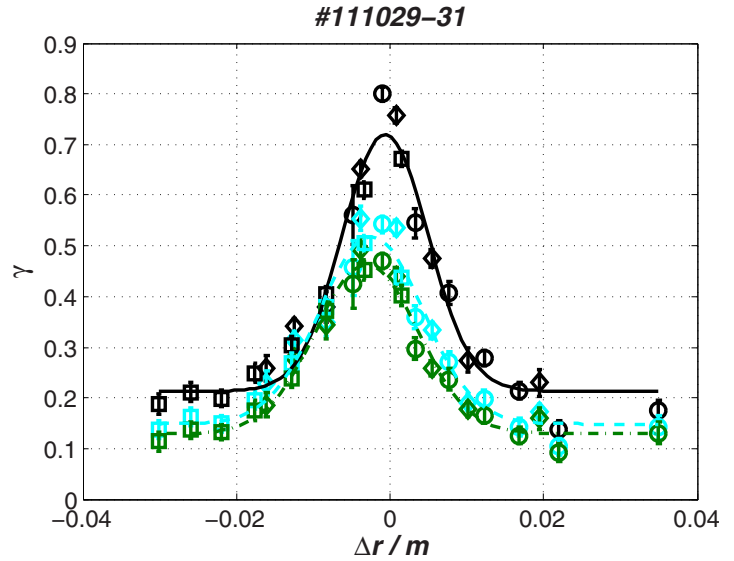

FIG. 9. (Color online) Measurement of the radial correlation length as a function of $\Delta \theta$ for the top antenna array. Different data symbols denote different discharges: solid line: $\Delta \theta=0.025 \mathrm{rad}$ (combination $\mathrm{E}, \mathrm{C}$ ), dashed line: $\Delta \theta=0.075 \mathrm{rad}$ (combination $\mathrm{D}, \mathrm{C}$ ), and dot-dashed line: $\Delta \theta=0.1 \mathrm{rad}$ (combination $\mathrm{B}, \mathrm{C}$ ).

sity was chosen to be $\bar{n}_{e}=2.0 \times 10^{19} \mathrm{~m}^{-3}$. One of the generators was kept constant in frequency, whereas the other one varies the frequency in steps of $700 \mathrm{MHz}$ in the range of $25.3 \leq f_{2} \leq 30.2 \mathrm{GHz}$ during the flat top of the discharge. Each frequency step lasts for $400 \mathrm{~ms}$. To decrease the radial separation between data points each discharge was repeated three times for $f_{1}=26,28$, and $30 \mathrm{GHz}$. For the estimation of the reflection layer the electron density profiles from the $\mathrm{HCN}$-interferometer are used. The radial range of the measurements is determined as $0.81 \leq r / a \leq 0.88$. Since only one antenna is connected to the frequency scanning generator, three combinations with different poloidal separations $(\Delta \theta$ $=0.025,0.05$, and $0.075 \mathrm{rad})$ can be used. The estimated radial correlations are in fact a combination of radial and poloidal correlations. However, due to three different poloidal separations the radial correlation length can be extrapolated. In Fig. 9 the coherence for the ambient turbulence is presented as a function of the radial separation for the top antenna array. The different line styles denote different $\Delta \theta$ and the different symbols denote the data from discharges

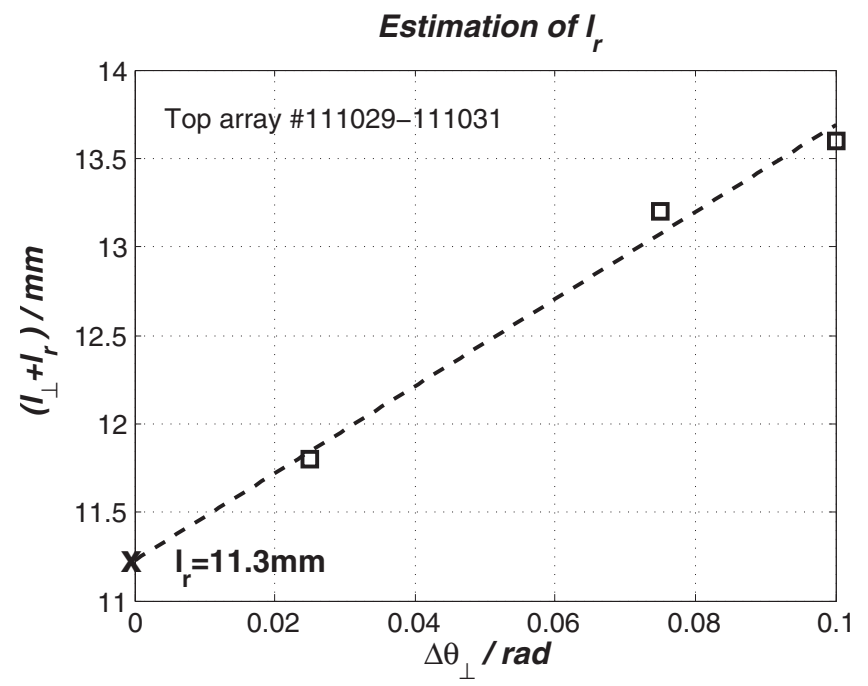

FIG. 10. Estimation of the radial correlation length $l_{r}$ for the top antenna array. 
with different $f_{1}$. As expected, the coherence drops with the increase of the radial and poloidal separation. The data for each value of $\Delta \theta$ can be approached by a Gaussian. The full width at $1 / e$-level of the Gaussian amplitude is a measure for the sum of radial and poloidal correlation length. These values are presented as a function of $\Delta \theta$ in Fig. 10 and yield a $l_{r}=11 \mathrm{~mm}$ at $\Delta \theta_{\perp}=0$. Similar values for $l_{r}$ are found from probe measurements at the plasma edge ${ }^{15}$ at TEXTOR. Furthermore the obtained $l_{r}$ is in the range of $l_{\perp} / 2 \leq l_{r} \leq l_{\perp}$, which was obtained in earlier measurements at TFTR. ${ }^{16}$ At TEXTOR the perpendicular correlation length is measured at the same time from combinations of antennas B, D, and E and yields $l_{\perp}=24 \mathrm{~mm}$.

\section{SUMMARY}

The paper discusses the strength of correlation reflectometry applied at the tokamak TEXTOR. With state of the art heterodyne reflectometers with phase locked loop, a large frequency band $(24-40 \mathrm{GHz}$ ) can be accessed by one reflectometer only. At TEXTOR, a sophisticated antenna setup consisting of three antenna arrays located at different poloidal and toroidal angles allows the measurement of turbulence properties and plasma rotation at the same time. Hereby, a second reflectometer is used to determine radial correlation length as well. Furthermore, the arrangement of the antennas in an array allows to deduce the pitch angle of the magnetic field line and therefore enables the measurement of the local measurement of the poloidal magnetic field and the safety factor $q$. First measurements of long range correlations for the geodesic acoustic mode show the large potential of correlation reflectometry. The measurement of the radial correlation length confirms an existing relation between radial and poloidal correlation lengths and it can be used for a detailed study of the radial correlation length profile in the vicinity of transport barriers.

\section{ACKNOWLEDGMENTS}

The authors like to thank the TEXTOR Team for operating the device and the support for installing all necessary in vessel components.

${ }^{1}$ Wikipedia-contributors, Stellerator (Wikipedia, The Free Encyclopedia, Date of last revision: 13 June 2010, 22:08 UTC), http://en.wikipedia.org/ w/index.php?title $=$ Stellarator\&oldid $=3 \% 67848849$.

${ }^{2}$ Wikipedia-contributors, Tokamak (Wikipedia, The Free Encyclopedia, Date of last revision: 10 June 2010, 13:47 UTC), http://en.wikipedia.org/ w/index.php?title $=$ Tokamak\&oldid $=36720 \% 3587$.

${ }^{3}$ R. Wolf, Plasma Phys. Controlled Fusion 45, R1 (2003).

${ }^{4}$ Wikipedia-contributors, ITER (Wikipedia, The Free Encyclopedia, Date of last revision: 6 June 2010, 17:13 UTC), http://en.wikipedia.org/w/ index.php?title $=$ ITER\&oldid $=36639631 \% 0$.

${ }^{5}$ T. L. Rhodes, R. J. Taylor, and W. A. Peebles, Rev. Sci. Instrum. 66, 824 (1995).

${ }^{6}$ V. Vershkov, V. Dreval, and S. Soldatov, Rev. Sci. Instrum. 70, 1700 (1999).

${ }^{7}$ S. Hacquin, L. Meneses, L. Cupido, N. Cruz, L. Kokonchev, R. Prentice, and C. Gowers, Rev. Sci. Instrum. 75, 3834 (2004).

${ }^{8}$ A. C. A. Figueiredo, A. Fonseca, and L. Meneses, Rev. Sci. Instrum. 79, 10F107 (2008).

${ }^{9}$ M. Gilmore, W. A. Peebles, and X. V. Nguyen, Rev. Sci. Instrum. 72, 293 (2001).

${ }^{10}$ G. J. Kramer, R. Nazikian, and E. Valeo, Rev. Sci. Instrum. 74, 1421 (2003).

${ }^{11}$ T. H. Stix, Waves in Plasmas (American Institute of Physics, New York, 1992).

${ }^{12}$ O. Neubauer, G. Czymek, B. Giesen, P. W. Hüttemann, M. Sauer, W. Schalt, and J. Schruff, Fusion Sci. Technol. 47, 76 (2005).

${ }^{13}$ A. Krämer-Flecken, S. Soldatov, H. R. Koslowski, and O. Zimmermann, Phys. Rev. Lett. 97, 045006 (2006).

${ }^{14}$ A. Fujisawa, Nucl. Fusion 49, 013001 (2008).

${ }^{15}$ Y. Xu, M. Van Schoor, R. R. Weynants, S. Jachmich, M. Vergote, M. W. Jakubowski, P. Beyer, M. Mitri, B. Schweer, D. Reiser, B. Unterberg, K. H. Finken, M. Lehnen, R. Jaspers, and the TEXTOR Team, Nucl. Fusion 47, 1696 (2007).

${ }^{16}$ R. Fonck, N. Bretz, G. Cosby, R. Durst, E. Mazzucato, R. Nazikian, S. Paul, S. Scott, W. Tang, and M. Zarnstorff, Plasma Phys. Controlled Fusion 34, 1993 (1992). 